\title{
On the development of a spherical hybrid model - Lessons and applications
}

\author{
S. Dyadechkin ${ }^{1,2}$, E. Kallio ${ }^{1}$, R. Jarvinen ${ }^{1}$, P. Janhunen ${ }^{1}$, \\ V. S. Semenov ${ }^{2}$ and H. K. Biernat ${ }^{3}$ \\ ${ }^{1}$ Finnish Meteorological Institute, Helsinki, Finland \\ P.O. BOX 503 FI-00101 Helsinki Finland \\ email: egopost@gmail.com \\ ${ }^{2}$ Saint-Petersburg State University, Russia \\ 198504, 1 Ulyanovskaya, Petrodvorets, St.-Petersburg, Russia \\ ${ }^{3}$ Space Research Institute Austrian Academy of Sciences \\ Schmiedlstrasse 6, 8042 Graz, Austria
}

\begin{abstract}
We are developing a spherical hybrid model to study how the solar wind interacts with the solar system bodies. In this brief status report we introduce some lessons from the spherical grid development and illustrate the usage of the new model by showing a preliminary test run.
\end{abstract}

Keywords. Solar wind, methods:numerical, stellar dynamics

\section{Introduction}

The solar wind plasma interacts with the different Solar System bodies in several ways. The different celestial objects reveal specific properties: The Moon does not have either an atmosphere or a global intrinsic magnetic field. Mercury does not have an atmosphere but it has a weak intrinsic magnetic field that forms a "pocket magnetosphere". Venus and Mars do not have a strong global intrinsic magnetic field but they have atmospheres dense enough to produce shields against the solar wind by their ionospheres. Martian magnetic anomalies can also be considered as "mini magnetospheres". Titan's dense atmosphere, in turn, can meet subsonic plasma flow.

A hybrid approach provides an efficient way to model how the cosmic plasma interacts with non-magnetized and magnetized planetary objects. In a hybrid model ions are considered as particles, while electrons form a massless, charge neutralizing fluid. The basic properties of the HYB hybrid model are described in Kallio \& Janhunen (2003). The HYB hybrid model family is being developed at the Finnish Meteorological Institute (FMI) during the last decade. The model has been used successfully to describe how the flowing plasma interacts with various solar system bodies such as Mercury, Venus, the Moon, Mars, Saturnian moon Titan and asteroids.

One geometrical limitation of the HYB model is, however, that it assumes cube shaped grid cells. In order to expand the usage of the HYB model we have initialized a project aiming to develop a spherical coordinate version of the model. A spherical grid would give us important advantages compared with a Cartesian grid, such as: 1) A good grid resolution, because the grid size decreases automatically near the obstacle (the planetary surface) and 2) Natural boundary conditions for the obstacle, because the planetary surface covers r-constant surface of the grid. However, the implementation of a spherical grid into the hybrid code also entails some challenges: first, a complexity of the interpolation on the spherical grid in comparison with the Cartesian, and second, pole problems. 


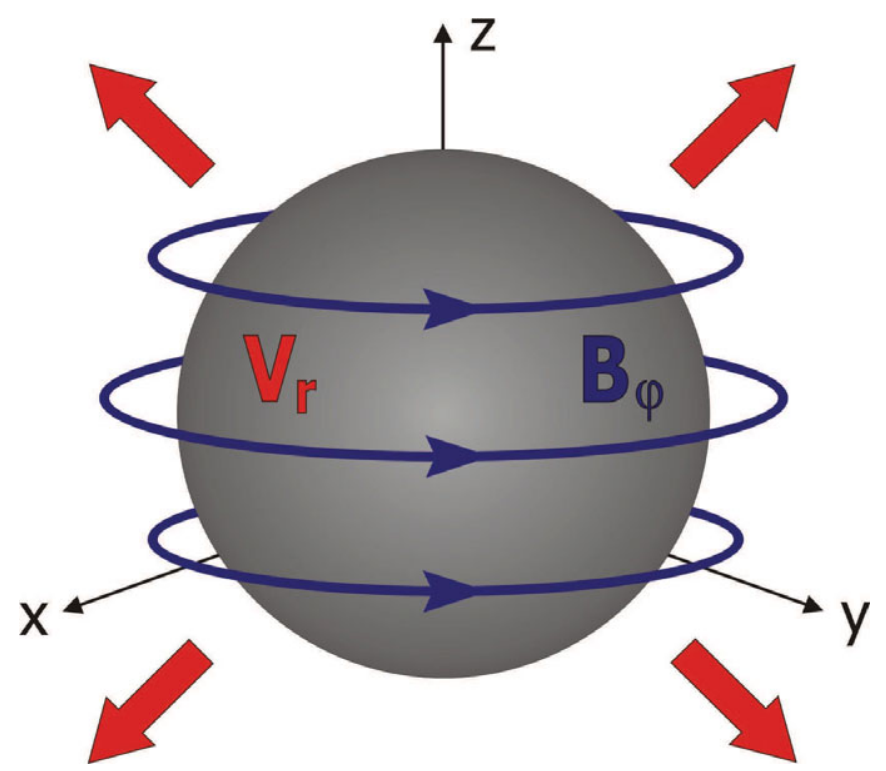

Figure 1. Initial conditions for the spherical expanding test. Initial magnetic field is posed on the spherical surface and it is purely toroidal (blue circles). Initial velocities of protons are perpendicular to the surface (red arrows).

\section{Spherical grid in a hybrid model}

In this section we briefly describe main advantages and challenges of spherical grid when it is used in a hybrid model:

\subsection{Advantages}

1 Spherical worlds. Planetary worlds are spherical and to simulate them it seems to be more natural to use a spherical grid instead of a Cartesian grid.

2 Grid resolution. In spherical coordinates there is "natural" grid refinement: the closer the cells are to the obstacle the smaller grid cells become. This property could be used for the introducing of a self-consistent ionosphere into the hybrid model, where we need to decrease the cell size at low altitudes.

3 Obstacle boundary condition. Another "natural" property of spherical coordinates is the geometrical interpretation of the obstacle. Planetary surface is covered by the $r=$ constant surface of the spherical grid, which simplifies the implementation of the boundary condition.

\subsection{Challenges}

1 Interpolations. Hybrid approach imply a number of vector and scalar value interpolations between different grid elements: interpolation from cell center to cell nodes (CN - interpolation), interpolation from cell faces to cell center (FC - interpolation) etc. As the spherical grid is not homogeneous, the realization of interpolation methods is not as straightforward as in the Cartesian coordinates.

2 Pole Problems. Spherical grid includes two singular points, poles, where the numerical values are not defined. Moreover, in circumpolar region numerical values "feel" singularities, because the spherical cell elements, edge lengths, face areas and cell volume become smaller when the cell is closer to the pole. There are several techniques to exclude the poles from the grid, most of them based on the decomposition of spherical grid into several grids (Usmanov, 1995; Kageyama \& Tetsuya, 2004; Ronchi et. al., 1996). 

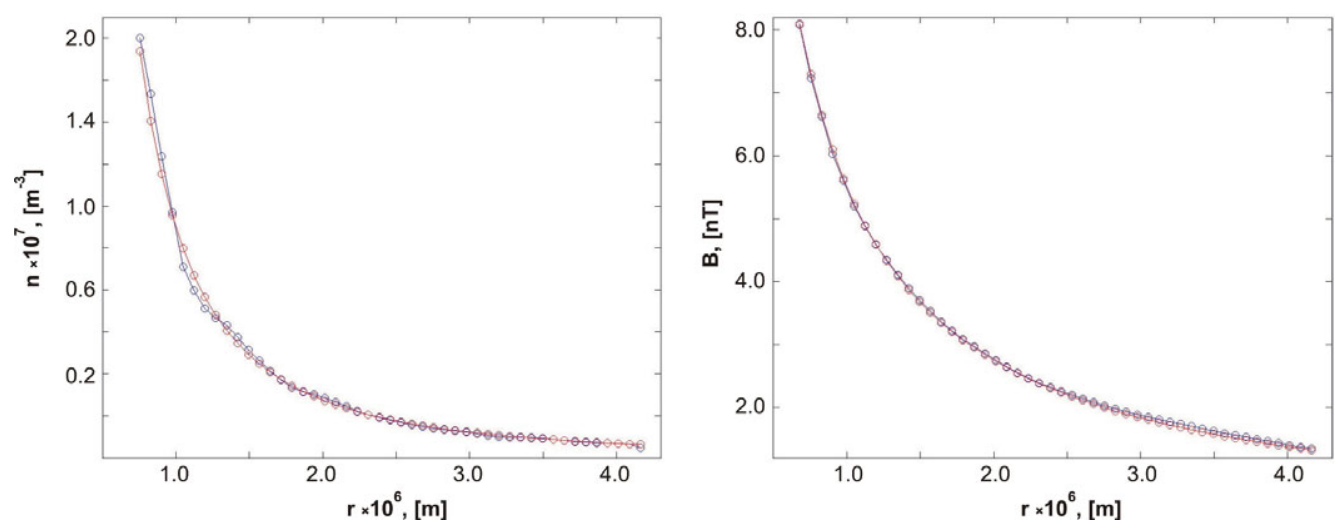

Figure 2. The figure shows analytical and experimental profiles of the number density and the magnetic field for a steady-state solution of a super-Alfvenic plasma flow from a spherical surface at $r=0.6 \cdot 10^{7} \mathrm{~m}$. Analytical curve is shown in red and experimental in blue. For the super-Alfvenic regime we can find an analytical approximation for the dependency of the number density and magnetic field on the radial distance. In the equatorial plane $(\theta=0)$ the number density is proportional to $1 / r^{2}$, and the magnetic field to $1 / r$.

\section{Spherical expanding test}

To illustrate preliminary results of the spherical hybrid model let's consider the superAlfvenic plasma flow from the spherical surface. The initial conditions of the spherical expanding test are depicted on Fig. 1.

Initial state: A spherical empty box and a magnetized wind from the spherical surface. Initially there is no plasma in the simulation box; only internal (obstacle) boundary conditions are defined.

Initial magnetic function: Initially there is only a toroidal component of the magnetic field $B=\left(0,0, B_{\phi}\right)$, which is defined as: $B_{\phi}=B_{0} \sin \theta$, where $B_{0}=1 n T$. To exclude pole problems from our simulation we used $\sin \theta$ dependence in the initial magnetic configutation.

Particle population: We consider only one $H^{+}$population with number density $n=$ $14 \cdot 10^{6} \mathrm{~m}^{-3}$ and temperature $T=10^{5} \mathrm{~K}$. The initial velocity has only a radial component $V=\left(V_{r}, 0,0\right)$, where $V_{r}=4 \cdot 10^{5} \mathrm{~m} / \mathrm{s}$. The average number of macroparticles per a cell is 30 .

\section{Summary}

A spherical hybrid model is anticipated to provide a powerful tool for the modeling of self-consistent cosmic plasma interactions with various solar system bodies and exoplanets. Developing such a model is, however, a challenging task bacause of the complexity of the interpolation and the polar region of the grid.

\section{References}

Kallio, E. \& Janhunen P. 2003, Annales Geophysicae, 21, 2133

Usmanov, A. V. 1993, Saint Petersburg State Univ., International Solar Wind 8 Conference, 65

Kageyama, A. \& Tetsuya, S. 2004, Geochemistry Geophysics Geosystems, 5, 9

Ronchi, C., Iacono, R., \& Paolucci, P. S. 1996, Journal of Computational Physics, 124, 1, 93 